\title{
Endostatin polymorphism 4349G/A(D104N) is not associated with aggressiveness of disease in postate cancer $^{1}$
}

\author{
He Cheng $\mathrm{Li}^{\mathrm{a}, * *}$, Qiu Yin Cai ${ }^{\mathrm{b}}$, Eric T. Shinohara ${ }^{\mathrm{c}}$, Hui Cai ${ }^{\mathrm{b}}$, Carolyn $\mathrm{Cao}^{\mathrm{c}}$, Zuo Fei Wang ${ }^{\mathrm{c}}$, \\ Ming Teng ${ }^{\mathrm{c}}$, Wei Zheng ${ }^{\mathrm{b}}$ and $\mathrm{Bo} \mathrm{Lu}^{\mathrm{c}, *}$ \\ ${ }^{a}$ Department of Breast Surgery, Cancer Hospital/Cancer Institute, Fudan University, Shanghai, 200032, P.R. China \\ ${ }^{\mathrm{b}}$ Department of Medicine and Vanderbilt-Ingram Cancer Center, Vanderbilt University School of Medicine, \\ Nashville, TN 37232, USA \\ ${ }^{\mathrm{c}}$ Department of Radiation Oncology and Vanderbilt-Ingram Cancer Center, Vanderbilt University School of \\ Medicine, Nashville, TN 37232, USA
}

\begin{abstract}
Endostatin is an important inhibitory molecule which mediates the sequential steps involved in angiogenesis. Lower level or impaired function of endostatin is associated with a higher risk of developing malignant solid tumors and with a worse prognosis of the disease. The endostatin $\mathrm{N} 104$ polymorphism might be associated with an impaired ability to inhibit angiogenesis. We analyzed the tissues from 98 Caucasian prostate cancer patients for the presence of D104N polymorphism. The frequencies of homozygous $4349 \mathrm{G} / \mathrm{G}(104 \mathrm{D} / \mathrm{D})$, and heterozygous $4349 \mathrm{G} / \mathrm{A}(104 \mathrm{D} / \mathrm{N})$ were $83.67 \%(82 / 98)$ and $16.33 \%(16 / 98)$, respectively; no individuals were homozygous 4349A/A(104N/N). With the Fisher's exact test we found the genotype of D104N was not significantly related to age, tumor grade, PSA and clinical stage $(P>0.05)$. There was no difference in relapse free survival(RFS) or overall survival(OS) between patients with $104 \mathrm{D} / \mathrm{N}$ and those with $104 \mathrm{D} / \mathrm{D}(P=0.8283,0.3713$ respectively). We concluded that endostatin polymorphism was not associated with the aggressiveness of prostate cancer in Caucasian patients.
\end{abstract}

Keywords: Prostate cancer, endostatin, polymorphism

\section{Introduction}

The latest estimates of global cancer incidence show that prostate cancer has become the third most common cancer in men, with half a million new cases each year, almost $10 \%$ of all cancers in male [1-4]. Prostate cancer is usually regarded as a slow-growing tumor, and only $2.9 \%$ actually will die of the disease [5]. In order to

*Address for correspondence: Bo Lu, M.D., Ph.D., Department of Radiation Oncology and Vanderbilt-Ingram Cancer Center, Vanderbilt University School of Medicine, Nashville, TN 37232, USA. Fax.: +1 615343 0161; E-mail: Bo.Lu@vanderbilt.edu.

**Visiting research fellow at Vanderbilt University School of Medicine, Nashville, TN 37232, USA.

${ }^{1}$ This work is supported in part by DOD PC031161(BL), RO1CA90899(WZ) and UICC fellowship ICRETT1025(HL). better design therapeutic strategies for individual cases and identifying patients who would benefit from more vigilant surveillance, it would be of interest to study markers which can predict the outcome of disease. Angiogenesis, or the formation of new blood vessels from preexisting endothelium, is a fundamental step in tumor progression and metastasis [6,7]. Endostatin, a $\mathrm{Mr}$ 20,000 cleavage product of the $\mathrm{COOH}$-terminal domain of collagen XVIII(NC1), has been considered as an important inhibitory molecules that can medicate the sequential steps involved in angiogenesis. Higher serum levels of endostatin induced experimentally in mice and rats seem to cause regression of various types of solid tumors, including prostate cancer [8-10]. In addition, Down's syndrome patients, who have a higher serum levels of endostatin because of their three copies of the 
Table 1

Associations of endostatin polymorphisms with other clinical and pathological parameters

\begin{tabular}{cccc}
\hline \multirow{2}{*}{ Factors } & \multicolumn{2}{c}{4349 polymorphism } & \multirow{2}{*}{$P$ value } \\
\cline { 2 - 3 } & $4349 \mathrm{G} / \mathrm{G}(104 \mathrm{D} / \mathrm{D}) \mathrm{n}(\%)$ & $4349 \mathrm{G} / \mathrm{A}(104 \mathrm{D} / \mathrm{N}) \mathrm{n}(\%)$ & \\
\hline Age (y) & $30(88.24)$ & & \\
$\leqslant 60$ & $42(80.77)$ & $10(11.76)$ & 0.6886 \\
$60-70$ & $10(83.33)$ & $2(16.67)$ & \\
$>70$ & & & \\
PSA & $12(70.59)$ & $5(29.41)$ & 0.2791 \\
$\leqslant 4$ & $45(86.54)$ & $7(13.46)$ & \\
$4-10$ & $25(86.21)$ & $4(13.79)$ & \\
$>10$ & & & 0.2640 \\
Gleason grade & $54(87.10)$ & $8(12.90)$ & \\
$\leqslant 6$ & $28(77.78)$ & $8(22.22)$ & \\
$>6$ & & & \\
Clinical stage & $7(77.78)$ & $2(22.22)$ & \\
I & $57(85.07)$ & $10(14.93)$ & \\
II & $18(81.82)$ & $4(18.18)$ & \\
III & &
\end{tabular}

COL18A1 gene, have a decreased incidence of solid tumors, including prostate cancer [11]. Thus, lower levels or an impaired function of endostatin might be associated with a higher risk of developing malignant solid tumor or with a worse prognosis of the disease. Visakorpi et al. conducted a systematic analysis of the COL18A1 gene and found a missense mutation of D104N located in the $\mathrm{COOH}$-terminal globular domain $\mathrm{NC} 1$ of collagen XVIII, the encoding region of endostatin [12]. Iughetti et al. found that heterozygous N104 individuals have a higher chance of developing prostate cancer compared with homozygous D104 subjects and proposed the presence of N104 impairs the function of endostatin [13]. In the present study we hypothesize that N104 may impair the function of endostatin and in turn affect the aggressiveness of prostate cancer. To testify our hypothesis we assessed the endostatin polymorphism present in 98 Caucasian patients with prostate cancer to determine whether D104N is correlated with well known prognostic factors as well as relapse free survival(RFS) and overall survival(OS).

\section{Materials and methods}

\subsection{Patient population and clinical data}

The study population consisted of 98 Caucasian patients with prostate cancer who underwent prostatectomy at Vanderbilt Hospital in 1997. The study was approved by Institutional Review Board (IRB\# 030986) at Vanderbilt University School of Medicine. All patients had clinically localized prostate cancer and were treated with radical prostatectomy as a primary treat- ment. All patients had adenocarcinoma confirmed histologically. The patients were followed at Vanderbilt Hospital or at local hospitals with a mean follow-up of 62 months. Patients' age ranged 40 to 81 with a median age of 63 years. All pathological information was reviewed by one pathologist and the tumor differentiation was evaluated using Gleason's score criteria. Clinical stage was classified according to the AJCC TNM staging system [14]. The Clinical and histological characteristics of the patients are summarized in Table 1.

\subsection{Tissue preparation and DNA extraction}

Using a standard microtome with disposable blades, 5 um thickness sections of a representative areas of normal prostatic glands were cut from the paraffin embedded blocks and stained with Hematoxylin and eosin (H\&E) and then examined under a microscope to verify the absence of prostate cancer. 5 um thickness section (about $1 \mathrm{ug}$ ) from each patient was used for DNA extraction. The sections were deparaffinized with xylene at room temperature for 30 minutes twice. Then the deparaffinized tissue was washed with $100 \%$ ethanol twice. After the ethanol had evaporated completely, the tissue was completely lysed with proteinase K. Then QIAamp DNA Mini Kit (QIAGEN Inc, Valencia, CA) was used to extract extraction and purify DNA from the tissues according to the tissue protocol of the kit.

\section{Polymorphism genotyping}

The allelic discrimination of the endostatin gene 4349G/A polymorphism was assessed with the ABI 


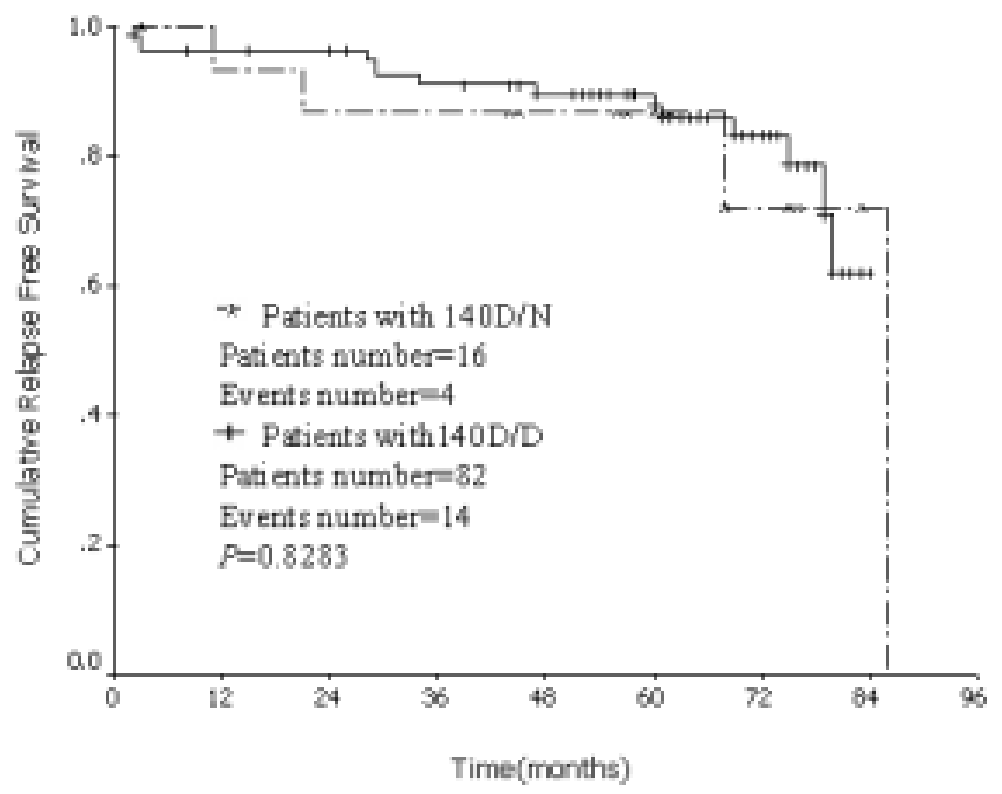

(A)

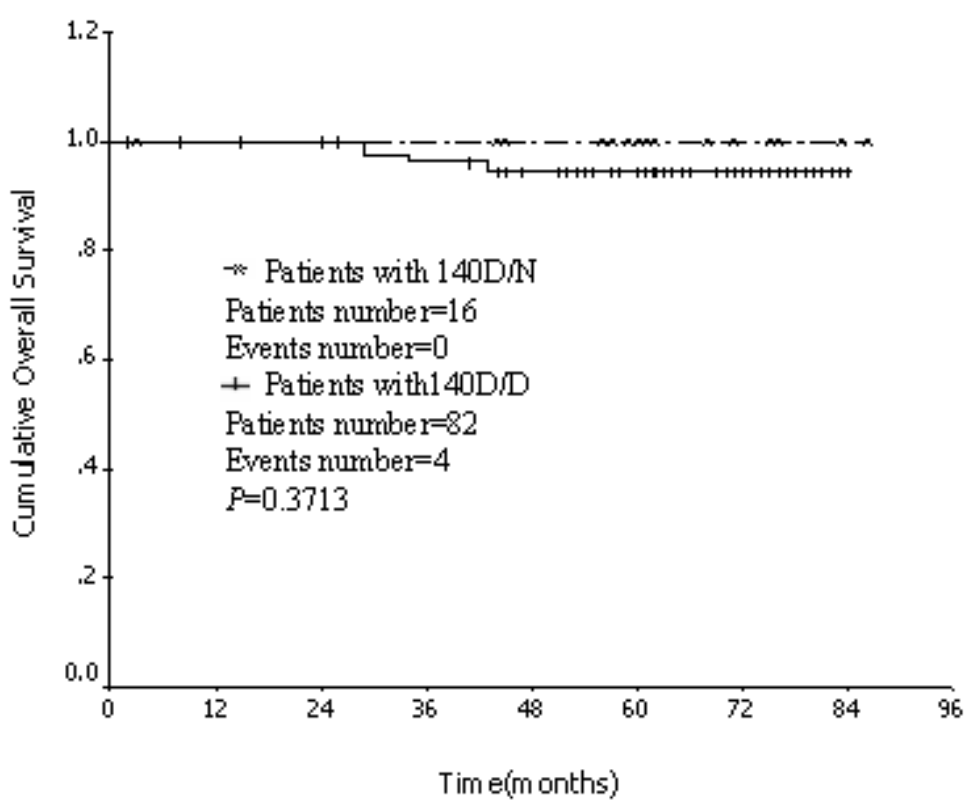

(B)

Fig. 1. Relapse free survival and overall survival curves. (A) RFS curves for patients with 4349G/G(140D/D) genotype and those with 4349G/A(140D/N); (B) OS curves for patients with 4349G/G(140D/D) genotype and those with 4349G/G (140D/N).

PRISM 7900 HT Sequence Detection System (Applied Biosystems). PCR was performed with a total volume of $5 \mathrm{ul}$, which contained approximately $2.5 \mathrm{ng}$ DNA, 1 $\times$ Taqman Universal PCR Master Mix, each primer at a concentration of $900 \mathrm{nM}$, and each probe at a concen- tration of $200 \mathrm{nM}$. Primers for endostatin were from Applied Biosystems (Assay ID: C_11872896_10). Taqman probes were: A allele specific: 5'-VIC-GCC CGG GGC ACG CAT CTT CTC CTT TAA CGG CAA GGA CGT CCT GAG GCA CCC -NFQ-3'.G-allele specific: 


\section{5'-FAM- GCC CGG GGC ACG CAT CTT CTC CTT} TGA CGG CAA GGA CGT CCT GAG GCA CCC -NFQ- $3^{\prime}$. The thermal cycling conditions were as follows: $95^{\circ} \mathrm{C}$ for $10 \mathrm{~min}$ to activate the AmpliTaq Gold enzyme, followed by 40 cycles of 15 seconds at $95^{\circ} \mathrm{C}$ and 60 seconds at $60^{\circ} \mathrm{C}$. The fluorescence levels were measured with an ABI PRISM 7900 HT Sequence Detector (Applied Biosystems, Foster City, CA), resulting in clear identification of three genotypes of each polymorphism.

The laboratory staff was blind to the identity of the subjects. Quality control (QC) samples were included in the genotyping assays. Each 384 well plate contained four water, eight CEPH 1347-02 DNA, eight blinded QC samples, and eight unblinded QC samples. The blinded and unblinded QC samples were taken from the second tube of study samples included in this study.

\section{Statistical analysis}

Relapses free survival (RFS) was defined as the time between the date of the primary surgery to the date of relapse or the date of last follow-up. Overall survival (OS) was defined as time between the date of the primary surgery to the date of death or the date of last follow-up. The Kaplan-Meier method was used to compute 5-year survival rates and log-rank test was applied to test the difference in survival across different genotype. The associations between the endostatin polymorphism and other clinical and pathological characters was analyzed by Fisher's exact test. P-values $<$ 0.05 were considered statistically significant. All statistical analyses were two sided. We conducted the statistical analysis with SAS 9.1 SAS Institute Inc., Cary, NC.

\section{Results}

\subsection{The correlation of $104 \mathrm{D} / \mathrm{N}$ polymorphism with other clinical and pathological parameters}

With Taqman SNP genotyping assay, the concordance of the blinded samples was $100 \%$. Genotypes for 4349G/A polymorphism were successfully determined in 98 samples. The frequencies of homozygous 4349G(104D/D), and heterozygous 4349G/A(104D/N) were $83.67 \%(82 / 98)$ and $16.33 \%(16 / 98)$, respectively. With Fisher's exact test, Endostatin polymorphism was found to have no significant relationship with age $(P=$ $0.6886)$, PSA $(P=0.2791)$, Gleason's grade $(P=$ $0.2640)$ and clinical stage $(P=0.7479)$ (Table 1$)$.

\subsection{Survival analysis}

The Kaplan-Meier method was used to compute survival rates and the log-rank test was applied to test the difference in survival across different genotypes. 5year relapse free survival rates and overall survival rates for this group of patients were $87.71 \%$ and $95.64 \%$ respectively. There was no significant difference between subjects with D104N and those with D104D in RFS and OS (Log-rank test, $P=0.8283$ and 0.3713 respectively) (Fig. 1A,B).

\section{Discussion}

In the current study, we analyzed the $4349 \mathrm{G} / \mathrm{A}$ (D104N) polymorphism in 98 Caucasian patients and did not find any association between the polymorphism and other clinical and pathological variables, and we did not find any significant association between 4349G/A(D104N) polymorphism and patients' survival (RFS and OS) either.

Higher serum levels of endostatin induced experimentally in mice and rats seem to cause regression of various types of solid tumors, including prostate cancer [8-10]. Clinical study also showed that individuals with higher levels of endostatin might be less prone to develop solid tumors [15]. The missense mutation of D104N located in the COOH-terminal globular domain NC1 of collagen XVIII was found to be in the encoding region of endostatin by Visakorpi et al. in 1999 [12]. It has been recently shown that gene polymorphisms affect cancer cell proliferation, differentiation as well as tumor invasion/metastasis [16]. The possible association of D104N with cancer risk and prognosis remains poorly investigated. Iughetti et al reported that individuals heterozygous for N104 have a 2.5 times greater chance of developing prostate cancer as compared with homozygous D104 subjects (OR: 2.4, 95\% CI:1.4-4.16); the author proposed that endostatin N104 has a decreased affinity for binding to other molecules and an impaired ability to inhibit angiogenesis [13]. In a case-control study containing 58 multiple myeloma patients, Ortega et al reported that the genotype frequencies were in Hardy-Weinberg equilibrium and that the $\mathrm{D} 104 \mathrm{~N}$ polymorphism was an unimportant determinant in multiple myeloma patients [17]. Liu et al. reported a case control study including 274 patients with leukemia and 178 normal controls. In Liu's study there were no patients homozygous for 4349A which was similar to our study; they found no association with 
endostatin polymorphism and the risk of leukemia $[17$, 18]. Most of the previous studies showed there were no association between the D104N polymorphism and malignancies. Only Iughetti et al. reported a higher risk ratio of developing prostate cancer for heterozygous N104 individuals, but even in this study they did not find any association between the presence of the mutation and Gleason score or age at diagnosis. We not only further confirm D104N polymorphism had no relationship with Gleason score or age at diagnosis, but also demonstrate that the D104N polymorphism has no relationship with PSA level, clinical stage as well as RFS and OS of patients.

The discrepancies between our study and previous studies might due to several reasons. The translation of results from different studies might be limited due to the limited sample size and different patient selection. In our study we selected stage I or II Caucasian patients. Another reason is that we analyze the relationship between the endostatin polymorphism and prostate cancer from the aspect of aggressiveness of disease but not cancer risk and this will also cause the discrepancies between our study and previous ones. Last but not the least reason, most of the previous studies as well as ours were retrospective and the conclusions must be drawn cautiously. So far, to the authors' knowledge there is no data from prospective randomized study available.

In conclusion, our study indicated that the endostatin polymorphism 4349G/A(D104N) is not associated with aggressiveness of disease in Caucasian patients with prostate cancer.

\section{Acknowledgments}

This study was supported in part by DOD PC031161 (BL), RO1CA90889(WZ) and UICC fellowship ICRETT1025(HL). We are grateful to Regina Courtney (Department of Medicine and Vanderbilt-Ingram Cancer Center, Vanderbilt University School of Medicine, Nashville, TN 37232, USA) for her technique support in Taqman SNP genotyping assay.

\section{References}

[1] D. Parkin, Royal College of Nursing, Momentum, Nurs Stand 452 (1986), 1-4.
[2] D.M. Parkin, E. Laara and C.S. Muir, Estimates of the worldwide frequency of sixteen major cancers in 1980, Int J Cancer 41 (1988), 184-197.

[3] D.M. Parkin, P. Pisani and J. Ferlay, Estimates of the worldwide incidence of 25 major cancers in 1990, Int J Cancer 80 (1999), 827-841.

[4] M. Quinn and P. Babb, Patterns and trends in prostate cancer incidence, survival, prevalence and mortality, Part I: international comparisons, BJU Int 90 (2002), 162-173.

[5] J.A. Siegal, E. Yu and M.K. Brawer, Topography of neovascularity in human prostate carcinoma, Cancer 75 (1995), 25452551.

[6] D. Hanahan and J. Folkman, Patterns and emerging mechanisms of the angiogenic switch during tumorigenesis, Cell $\mathbf{8 6}$ (1996), 353-364.

[7] T. Sasaki, N. Fukai, K. Mann, W. Gohring, B.R. Olsen and R. Timpl, Structure, function and tissue forms of the C-terminal globular domain of collagen XVIII containing the angiogenesis inhibitor endostatin, Embo J 17 (1998), 4249-4256.

[8] Y. Yokoyama, J.E. Green, V.P. Sukhatme and S. Ramakrishnan, Effect of endostatin on spontaneous tumorigenesis of mammary adenocarcinoma in a transgenic mouse model, Cancer Res 60 (2000), 4362-4365.

[9] S.S. Yoon, H. Eto, C.M. Lin, H. Nakamura, T.M. Pawlik, S.U. Song and K.K. Tanabe, Mouse endostatin inhibits the formation of lung and liver metastases, Cancer Res 59 (1999), 6251-6256.

[10] G. Perletti, P. Concari, R. Giardini, E. Marras, F. Piccinini, J. Folkman and L. Chen, Antitumor activity of endostatin against carcinogen-induced rat primary mammary tumors, Cancer Res 60 (2000), 1793-1796.

[11] H. Hasle, I.H. Clemmensen and M. Mikkelsen, Risks of leukaemia and solid tumours in individuals with Down's syndrome, Lancet 355 (2000), 165-169.

[12] T. Visakorpi, Molecular genetics of prostate cancer, Ann Chir Gynaecol 88 (1999), 11-16.

[13] P. Iughetti, O. Suzuki, P.H. Godoi, V.A. Alves, A.L. Sertie, T. Zorick, F. Soares, A. Camargo, E.S. Moreira, C. di Loreto, C.A. Moreira-Filho, A. Simpson, G. Oliva and M.R. PassosBueno, A polymorphism in endostatin, an angiogenesis inhibitor, predisposes for the development of prostatic adenocarcinoma, Cancer Res 61 (2001), 7375-7378.

[14] Prostate, in: American Joint Commitee on Cancer: AJCC Cancer Staging Manual, 6 eds, Springer, New York, 2002, pp. 309-316.

[15] O. Musso, M. Rehn, N. Theret, B. Turlin, P. Bioulac-Sage, D. Lotrian, J.P. Campion, T. Pihlajaniemi and B. Clement, Tumor progression is associated with a significant decrease in the expression of the endostatin precursor collagen XVIII in human hepatocellular carcinomas, Cancer Res 61 (2001), 45-49.

[16] A. Loktionov, Common gene polymorphisms, cancer progression and prognosis, Cancer Lett 208 (2004), 1-33.

[17] M.M. Ortega, H. Nascimento, F.F. Costa, M.T. Teori and C.S. Lima, A polymorphism in the angiogenesis inhibitor, endostatin, in multiple myeloma, Leuk Res 27 (2003), 93-94.

[18] T.C. Liu, C.T. Peng, S.F. Lin, C.S. Chang, T.P. Chen and J.G. Chang, Assocation of endostatin D104N with leukemia, Kaohsiung J Med Sci 19 (2003), 1-5. 


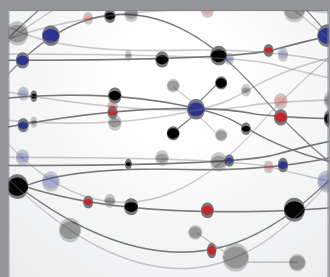

The Scientific World Journal
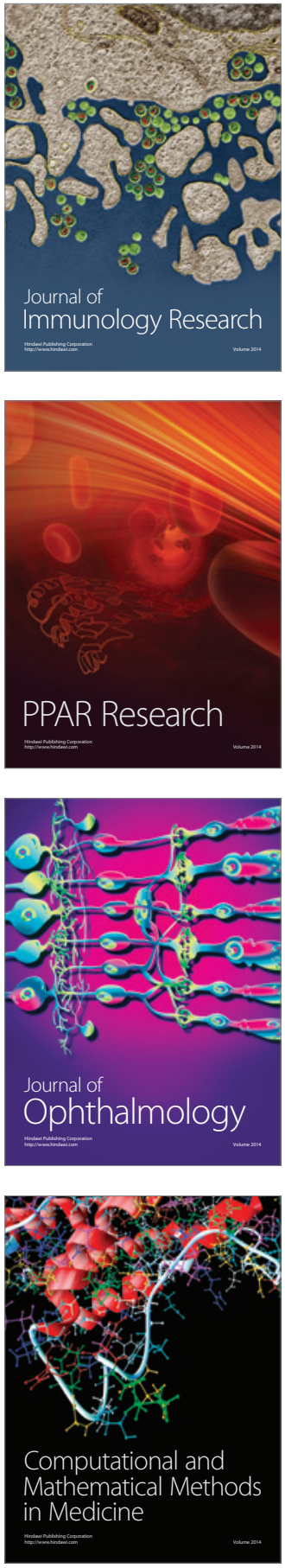

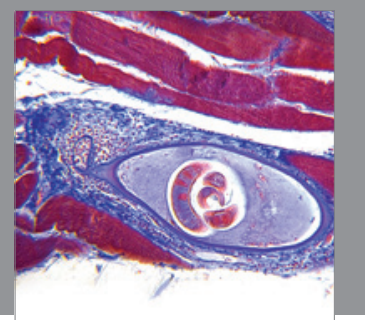

Gastroenterology

Research and Practice
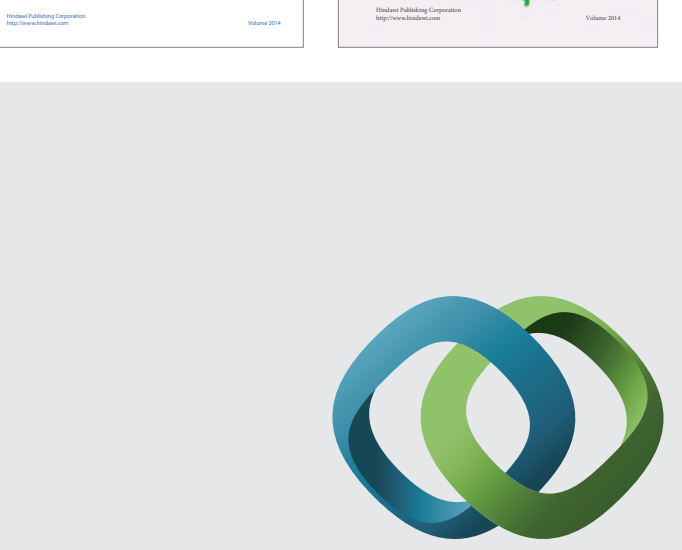

\section{Hindawi}

Submit your manuscripts at

http://www.hindawi.com
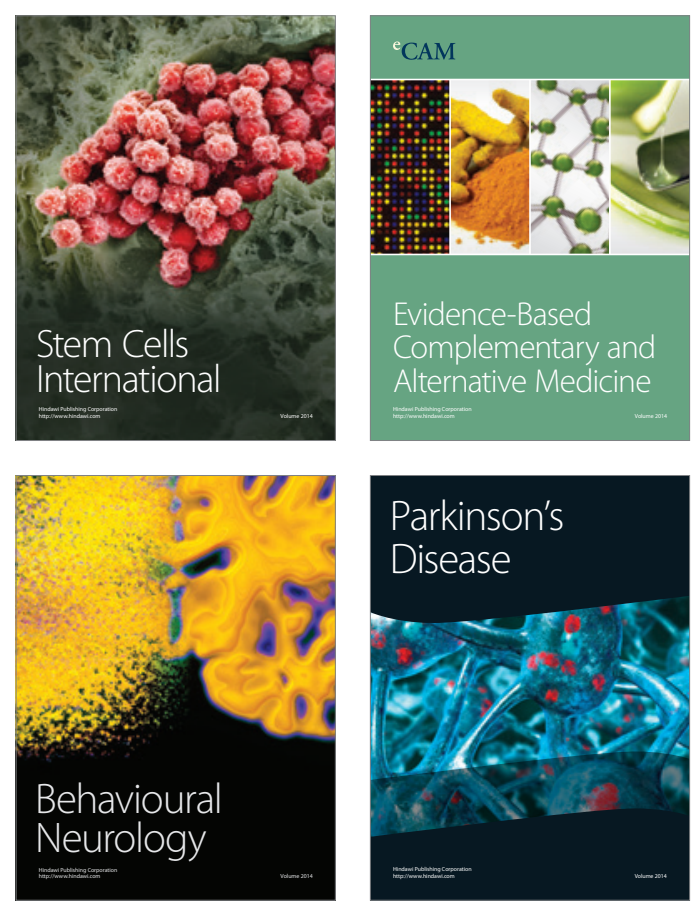

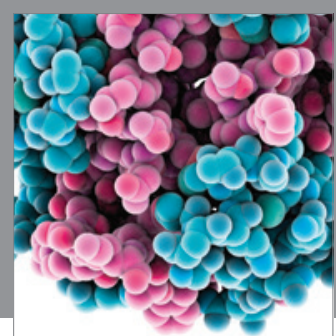

Journal of
Diabetes Research

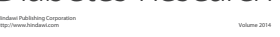

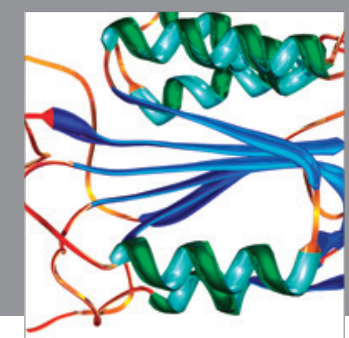

Disease Markers
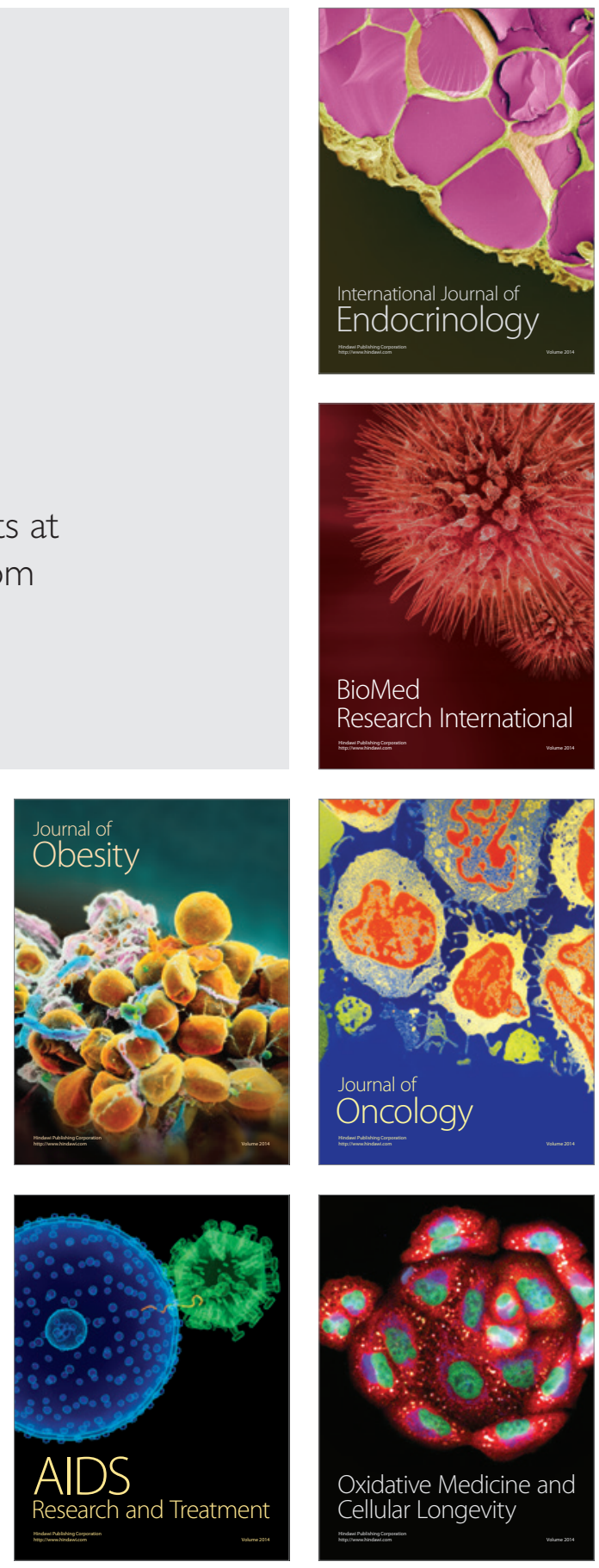